\title{
Thermal instability of an expanding dusty plasma with equilibrium cooling
}

\author{
Madhurjya P. Bora and Manasi Buzar Baruah \\ Physics Department, Gauhati University, Guwahati 781014, India.
}

\begin{abstract}
We present an analysis of radiation induced instabilities in an expanding plasma with considerable presence of dust particles and equilibrium cooling. We have shown that the equilibrium expansion and cooling destabilize the radiation condensation modes and the presence of dust particles enhances this effect. We have examined our results in the context of ionized, dusty-plasma environments such as those found in planetary nebulae (PNe). We show that due to the non-static equilibrium and finite equilibrium cooling, small-scale localized structures formed out of thermal instability, become transient, which agrees with the observational results. The dust-charge fluctuation is found to heavily suppress these instabilities, though in view of non-availability of convincing experimental data, a definitive conclusion could not be made.
\end{abstract}




\section{INTRODUCTION}

Thermal instability is long thought to be a relevant astrophysical process which is responsible for existence of smaller, non-gravitational condensations such as those found in solar prominences, interstellar clouds, and planetary nebulae $(\mathrm{PNe})$. Earlier works on thermal instability include those of Parker ${ }^{1}$, Weymann ${ }^{2}$, and Field ${ }^{3}$, among which, Field's seminal work on thermal instability is considered to be a comprehensive treatise on the subject till date. Recently, Gomez-Pelaez and Moreno-Insertis 4 have addressed the process of thermal instability in a gravitating medium undergoing expansion. Several other authors have considered occurrence of thermal instability in different contexts and parameter regimes. An analysis of the thermal instability of an optically thin plasma in the nonlinear regime is considered by Steele et. al..$^{\frac{5}{2}}$ Some of the very recent works involving numerical simulations, address the issues of dense structure formation in a hot protogalactic environment $\underline{\underline{6}} \underline{\underline{7}}$ and the interplay between thermal and magnetorotational instability (MRI) in interstellar media ${ }^{8,9}$.

It is also now well known that the dust particles constitute an ubiquitous and important component of many astrophysical plasmas including interstellar clouds, stellar and planetary atmospheres, planetary nebulae, giant H-II regions (GHR), hot interstellar matter (ISM), etc. The presence of dust in an ionized astrophysical structure, besides being responsible for introduction of new wave modes related to the dust dynamics, can also significantly modify the thermal structure of the plasma ${ }^{10,11}$. One of the important ways dust can modify the stability properties is by capturing energetic electrons and cooling through radiation 12,13 in the infrared region and we expect that the presence of dust particles modify the corresponding growth rates significantly. The charge fluctuation dynamics of the dust particles is also found to introduce new acoustic modes in the plasma ${ }^{14,15}$. Besides, charging of the dust grains makes them an integral part of the multi-component plasma which also helps maintain the colloidal nature of the plasma. Apart from the physical influence of the presence of dust particles in such astrophysical environments, the scenario becomes more complicated when one considers the dynamic behavior of the background. For example, the planetary nebulae are known to have severe equilibrium expansions, in many cases with supersonic expansion velocities $\frac{16}{217}$. It is therefore of particular interest to review the process of condensations in ionized plasmas taking into consideration the effect of the dust dynamics and their participation in the overall stability with a non-static background. In this work, we 
have undertaken a systematic study of the thermal condensation process in such an ionized plasma environment which undergoes equilibrium expansion and cooling having a considerable presence of dust particles which constitute another charged species of the plasma. We would like to view the outcome of this analyses in the context of condensations observed in planetary nebulae $(\mathrm{PNe})$.

The problem of existence of dust particles inside the hot ionized environment of a PNe is yet to be understood fully $\stackrel{18}{\underline{n}}$. It has however been proposed that majority of the dust grains could be destroyed in the hot ionized regions of plasma in a PNe, as it evolves, either because of spallation by hard UV photons or shocks. Subsequently, a PNe or a GHR should be free of dust grains $\frac{19,20}{2}$. However, in many cases the destruction of dust particles inside an ionized environment is not complete. For example, recent observations of the infrared spectra of the evolved nebula NGC $6445^{21,22}$, it has been shown that only a little destruction

of the dust grains inside the ionized region of the nebula could have occurred and dust-grain separation inside the ionized region is not plausible ${ }^{23}$. Even earlier attempts to detect Ca lines in PNes suggest that $\mathrm{Ca}$ is probably locked in the dust grains present inside the ionized environment 44,25 . Theoretical investigations, so far, indicate that dust particles are gradually accelerated to higher and higher velocities by the radiation pressure and are being expelled from a nebula 26,27 , which however do not take into account the mechanism of charging of the dust grains. The charging of the dust grains freezes them into the ionized gas $\frac{18}{}$, about which we have already referred.

In Section 2, we describe our model equations with background cooling and expansion. In Section 3, we apply the linear perturbation theory to examine the thermal condensation modes. In Section 4, we consider the case of static dust charge with the help of a WKB formalism and derive the linear dispersion relation. We numerically examine the dust-charge fluctuation dynamics in Section 5. Finally, we summarise our observations in Section 6.

\section{MODEL EQUATIONS}

Below, we write down the equations for a weakly collisional, fully ionized dusty plasma with an expanding background. The equations are namely, the equations of continuity, momentum, and energy conservation,

$$
\frac{\partial n_{j}}{\partial t}+\nabla \cdot\left(n_{j} \boldsymbol{v}_{j}\right)=0
$$




$$
\begin{aligned}
m_{j} n_{j}\left(\frac{\partial \boldsymbol{v}_{j}}{\partial t}+\boldsymbol{v}_{j} \cdot \nabla \boldsymbol{v}_{j}\right) & =-\nabla p_{j}-e n_{j} \nabla \phi-m_{j} n_{j} \nabla \psi, \\
\frac{3}{2} n_{j} \frac{d T_{j}}{d t}+p_{j} \nabla \cdot \boldsymbol{v}_{j} & =\chi_{j} \nabla^{2} T_{j}-m_{j} n_{j} \mathcal{L}_{j}\left(n_{j}, T_{j}\right),
\end{aligned}
$$

where the subscript $j=e$ and $d$ for the electrons and dust particles, $\chi_{j}$ is the thermal conductivity of the species, and $\phi$ and $\psi$ are the electrostatic and gravitational potentials defined by their respective Poisson's equations,

$$
\begin{aligned}
\nabla^{2} \phi & =-4 \pi\left[e\left(n_{i}-n_{e}\right)+Q n_{d}\right] \\
\nabla^{2} \psi & =4 \pi G m_{d} n_{d}
\end{aligned}
$$

$Q$ being the total charge on the dust particles. The ratio of specific heats $\gamma=5 / 3$ in these equations and other symbols have their usual meanings. As mentioned before, we have assumed the massive dust particle to be negatively charged. The second term on the right hand side of Eq.(3) represents the net heat-loss through radiation ${ }^{3}$. Note that, in writing these equations, we have assumed that the ions are Boltzmanian,

$$
n_{i}=n_{0} e^{-\frac{e \phi}{T_{i}}}
$$

This assumption of thermalization of ions comes from the fact that at temperature above $\sim 10^{4}{ }^{\circ} \mathrm{K}$, it is effectively the electron energy, which gets radiated and the radiative cooling of the electrons prevents them from attaining thermal equilibrium $\underline{15}, 28$. Besides, as we are

interested in the region of perturbation with phase velocity of the order of $\sim \sqrt{T_{e} / m_{d}}$; the ion thermal velocity $\left(\sim \sqrt{T_{e} / m_{i}}\right)$ can be assumed to be much higher, so that any temperature fluctuation in the ions are assumed to be quickly equilibrated. The pressures for the electrons and dust particles are given by $p_{j}=n_{j} T_{j}$. In what follows, we however neglect self-gravity, as the regimes where our analysis might be applicable are not sufficiently massive to be affected by self-gravity 29 .

\section{A. Background (equilibrium) expansion with net cooling}

We consider now a homogeneous and uniformly expanding background and assume that self-gravity is too weak to affect the expanding plasma so that the equilibrium is characterized by an expansion parameter $\varepsilon(t)^{\underline{4}, 30}$,

$$
\boldsymbol{r}=\varepsilon(t) \boldsymbol{x}, \quad \dot{\varepsilon}(t)=\text { const. }
$$


where $\boldsymbol{x}$ is the Lagrangian coordinate. Due to this non-static background, the equilibrium quantities naturally become time dependent. Note that when self-gravity is not negligible, the rate of expansion is not constant and is given by,

$$
\ddot{\varepsilon}=-\frac{4}{3} \pi G \rho_{0} \varepsilon
$$

where $\rho_{0}$ is equilibrium matter density. The equilibrium background velocity $\boldsymbol{v}_{0}$ is given by

$$
\boldsymbol{v}_{0}(\boldsymbol{r}, t)=\frac{\dot{\varepsilon}}{\varepsilon} \boldsymbol{r}
$$

This expanding equilibrium is mainly characterized by the equilibrium density $n_{j 0}$ and temperature $T_{j 0}$,

$$
\begin{aligned}
\frac{d n_{j 0}}{d t} & =-3 \frac{\dot{\varepsilon}}{\varepsilon} n_{j 0} \\
\frac{d}{d t} \log T_{j 0} & =-2\left(\frac{\dot{\varepsilon}}{\varepsilon}+\frac{1}{3} m_{j} \frac{\mathcal{L}_{j 0}}{T_{j 0}}\right),
\end{aligned}
$$

where the equilibrium quantities are denoted by a subscript ' 0 '. In the expression for evolution of equilibrium temperature Eq.(11), there are essentially two effects causing temperature to change - equilibrium background expansion [the first term in Eq.(11)] and net equilibrium cooling (the second term).

\section{THE LINEAR PERTURBATION ANALYSIS}

We perturb the equilibrium with a small electrostatic perturbation and for any arbitrary physical quantity $f(\boldsymbol{x}, t)$, we write the corresponding perturbation as

$$
f_{1}(\boldsymbol{x}, t) \sim f_{1}(t) e^{i \boldsymbol{k} \cdot \boldsymbol{x}}
$$

where $\boldsymbol{k}$ is the Lagrangian wave vector. In doing so, we have retained the time dependent part of the perturbation exclusively with the amplitude and Fourier decomposed the space part $\mathrm{B}^{30}$. We split the perturbed velocity into two components, namely parallel and perpendicular to $\boldsymbol{k}$,

$$
\boldsymbol{v}_{j 1}(t)=\frac{\boldsymbol{k}}{k} v_{j 1}(t)+\boldsymbol{v}_{j 1 \perp}(t)
$$

where $v_{j 1}(t)=\hat{\boldsymbol{k}} \cdot \boldsymbol{v}_{j 1}$. 
From the electron and dust continuity equations, the perturbed densities can be written as,

$$
\frac{d \hat{n}_{j}}{d t}=-i \tilde{k} v_{j 1}
$$

where the quantities with a "^' are perturbed quantities normalized by their equilibrium values and $\tilde{k}=k / \varepsilon$. If we neglect the inertia term of the electrons in the momentum equation, Eq.(2), compared to the massive dust particles, the perturbed electron pressure is just the perturbed electrostatic potential,

$$
\hat{p}_{e 1}=\frac{e \phi_{1}}{T_{e 0}}=\hat{\phi}
$$

in which, we have normalized the potential with the equilibrium electron temperature. Note that the temperature is expressed in energy units. As the ions are assumed to be Boltzmanian [Eq.([6)], the perturbed ion density can simply be expressed as,

$$
\hat{n}_{i}=-\tau \hat{\phi}
$$

where $\tau=T_{e 0} / T_{i o}$ is the ratio of the electron and ion equilibrium temperatures.

From the electron energy equation Eq.(3), we have,

$$
\begin{gathered}
\left(\frac{3}{2} n_{e} \frac{d T_{e}}{d t}+p_{e} \nabla \cdot \boldsymbol{v}_{e}\right)_{1}+\hat{p}_{e}\left(\chi_{0} \tilde{k}^{2} T_{e 0}+m_{e} n_{e 0} \mathcal{L}_{e 0}+m_{e} n_{e 0} T_{e 0}^{2} \mathcal{L}_{n_{e}}=0,\right. \\
-\hat{n}_{e}\left(\chi_{0} \tilde{k}^{2} T_{e 0}+m_{e} n_{e 0} T_{e 0}^{2} \mathcal{L}_{p_{e}}\right)
\end{gathered}
$$

where the ()$_{1}$ denotes the first order perturbation of the respective quantity and the equilibrium derivatives of the radiative loss function are defined as,

$$
\begin{aligned}
\mathcal{L}_{n_{e}} & =\left[\frac{\partial}{\partial T_{e 0}}\left(\frac{\mathcal{L}_{e 0}}{T_{e 0}}\right)\right]_{n_{e 0}}, \\
\mathcal{L}_{p_{e}} & =\left[\frac{\partial}{\partial T_{e 0}}\left(\frac{\mathcal{L}_{e 0}}{T_{e 0}}\right)\right]_{p_{e 0}} .
\end{aligned}
$$

The quantities inside the ()$_{1}$ are given as,

$$
\begin{aligned}
\left(p_{e} \nabla \cdot \boldsymbol{v}_{e}\right)_{1} & =p_{e 0}\left(\nabla \cdot \boldsymbol{v}_{e 1}\right)-\hat{p}_{e}\left[\frac{3}{2} n_{e 0} \frac{d T_{e 0}}{d t}+m_{e} n_{e 0} \mathcal{L}_{e 0}\right], \\
\left(n_{e} \frac{d T_{e}}{d t}\right)_{1} & =n_{e 0} \frac{d T_{e 1}}{d t}+n_{e 0}\left(\hat{p}_{e}-\hat{T}_{e}\right) \frac{d T_{e 0}}{d t}
\end{aligned}
$$

Finally using the perturbed electron continuity equation, Eq(14), we can write Eq.(17) for the perturbed electron temperature as,

$$
\frac{d \hat{p}_{e}}{d t}=-i \frac{5}{3} \tilde{k} v_{e 1}-\hat{p}_{e}\left(\tau_{\chi}^{-1}+\omega_{n}\right)+\hat{n}_{e}\left(\tau_{\chi}^{-1}+\omega_{p}\right)
$$


where $\tau_{\chi}, \omega_{n}^{-1}$, and $\omega_{p}^{-1}$ are the characteristic time-scales for the thermal conduction by the electrons, isochoric, and isobaric electron perturbations ${ }^{4}$, given by

$$
\tau_{\chi}^{-1}=\frac{2}{3} \frac{\chi_{0} \tilde{k}^{2}}{n_{e 0}}, \quad \omega_{n, p}=\frac{2}{3} m_{e} T_{e 0} \mathcal{L}_{n_{e}, p_{e}} .
$$

The quasi-neutrality condition with negatively charged dust particles is given by,

$$
\hat{n}_{e} \delta_{e}-\hat{n}_{i}+\left(\hat{n}_{d}+\hat{Z}_{d}\right) \delta_{d}=0
$$

where we have taken the possibility of finite dust-charge fluctuation. The quantities $\delta_{e}=$ $n_{e 0} / n_{i 0}$ and $\delta_{d}=n_{d 0} Z_{d 0} / n_{i 0}$ are the ratios of equilibrium electron and dust densities to the ion densities and $Q=q Z_{d}$ is the dust charge with $Z_{d}$ being the dust charge number. The dust charging equation is given by $\stackrel{14}{ }$,

$$
\frac{d Q_{d 1}}{d t}=I_{e 1}+I_{i 1}
$$

where, $I_{j 1} \mathrm{~s}$ are perturbed electron and ion currents. The above charging equation can be ultimately reduced to,

$$
\frac{d Q_{d 1}}{d t}+\eta Q_{d 1}=\left|I_{e 0}\right|\left(\frac{n_{i 1}}{n_{i 0}}-\frac{n_{e 1}}{n_{e 0}}\right)
$$

where $\eta$ is natural decay rate of charge fluctuations ${ }^{14}$.

Finally, we write the dust momentum equation Eq.(2) as,

$$
\frac{d v_{d 1}}{d t}=-\frac{\dot{\varepsilon}}{\varepsilon} v_{d 1}+i Z_{d 0} \omega_{d} c_{d} \phi_{1}
$$

where

$$
\omega_{d}=\tilde{k} c_{d}
$$

is the frequency of acoustic perturbation, and $c_{d}=\sqrt{T_{e 0} / m_{d}}$. By virtue of the non-static equilibrium, all the $\omega$ s i.e. $\omega_{n, p, d}$ have become time dependent quantities.

The complete closed set of equations for linear perturbation of our model is given by Eqs.(14) $-(16),(22),(24),(26)$, and (27) .

\section{A. Analysis of the perturbed system}

As the linearised differential equations become non-autonomous due to equilibrium expansion and net cooling, the problem, in general, is not amenable to standard Fourier decomposition for obtaining the dispersion relation, which requires rigorous numerical treatment as 
exact analytical solutions do not exist. However, depending on the time-scales of the problem, we can apply approximation techniques viz. WKB approximation ${ }^{4} 31$ to gain valuable insight into the nature of the problem. Recently Nejad-Asghar and Ghanbari ${ }^{32}$ have considered the problem of non-static equilibrium for radiation instability in molecular clouds, where they have used an ansatz of exponential form for the normalized perturbed quantities. In our notation, their formalism can be expressed as,

$$
\hat{n}_{d} \sim \frac{1}{\varepsilon(t)} e^{\omega t}
$$

where the quantity $\omega$ is analogous to normal mode frequency, which is decoupled from $t$. However, as the equilibrium is time dependent, the normal modes of the system become time dependent too, so that $\omega=\omega(t)$ and a decomposition of the kind expressed in Eq.(29) is not possible. As we show in the appendix, perturbation amplitude of densities based on Eq.(29) does not lead too far and grossly disagrees with the numerical analysis and the validity of the corresponding results are very limited.

In the following Section, we use the WKB approximation to analyse the perturbed system in the limit of static dust-charge.

\section{STATIC DUST CHARGE : WKB APPROXIMATION}

In order to simplify the mathematical complexity of the problem, we first consider the case of static dust charge. In this limit, we can reduce the relevant equations of Sec.II to form a third order differential equation in any of the perturbed variables, which can be conveniently expressed as,

$$
a_{3}(t) \frac{d^{3} \hat{n}_{d}}{d t^{3}}+a_{2}(t) \frac{d^{2} \hat{n}_{d}}{d t^{2}}+a_{1}(t) \frac{d \hat{n}_{d}}{d t}+a_{0}(t) \hat{n}_{d}=0
$$

where the coefficients $a_{i}(t) \mathrm{s}$ are given by,

$$
\begin{aligned}
& a_{3}(t)=3 \delta_{e}+5 \tau(t) \\
& a_{2}(t)=3 \delta_{e} \Omega_{n}+3 \tau(t) \Omega_{p}+6 \delta_{e} \omega_{c}+2 \omega_{\varepsilon}\left[9 \delta_{e}+10 \tau(t)\right] \\
& a_{1}(t)=2 a_{2}(t)-\omega_{\varepsilon}\left[9 \delta_{e}+10 \tau(t)\right]+5 \delta_{d} Z_{d 0}(t) \omega_{d}^{2} \\
& a_{0}(t)=3 \delta_{d} Z_{d 0}(t) \omega_{d}^{2} \Omega_{p}
\end{aligned}
$$


Various frequencies used in Eq.(30) are defined as

$$
\begin{aligned}
& \Omega_{n}=\omega_{n}(t)+\tau_{\chi}^{-1}(t), \\
& \Omega_{p}=\omega_{p}(t)+\tau_{\chi}^{-1}(t) .
\end{aligned}
$$

The expansion and cooling time-scales are given by $\omega_{\varepsilon, c}^{-1}$,

$$
\omega_{\varepsilon}=\left(\frac{\dot{\varepsilon}}{\varepsilon}\right), \quad \omega_{c}=\left(\frac{1}{3} m_{e} \frac{\mathcal{L}_{e 0}}{T_{e 0}}\right) .
$$

Different time-scales of the system are dictated by $\omega_{n, p, c, \varepsilon, \chi}^{-1}$, a clear separation of which is essential for WKB approximation.

\section{A. Linear dispersion relation}

Going by the standard WKB theory ${ }^{31}$, we expand the eigenfunctions $\hat{n}_{d}(t)$ of Eq.(30) as,

$$
\hat{n}_{d}(t) \sim \exp \left[\frac{1}{\delta} \sum_{n=0}^{\infty} \delta^{n} S_{n}(t)\right], \quad \delta \rightarrow 0,
$$

where $\delta$ is a small parameter and $S_{i}(t)$ are arbitrary functions of time. We break up the coefficients $a_{i}(t) \mathrm{s}$ in time as,

$$
a_{i}(t) \equiv a_{i}^{(0)}+a_{i}^{(1)} \epsilon^{-1}+a_{i}^{(2)} \epsilon^{-2}+\cdots
$$

where $\epsilon$ is the smallest time-scale of the system. We assume a-priori that the different coefficients $a_{i}(t)$ s can be ordered as,

$$
\begin{aligned}
& a_{3}(t)=a_{3}^{(0)}, \\
& a_{2}(t)=a_{2}^{(0)}+a_{2}^{(1)} \epsilon^{-1}, \\
& a_{1}(t)=a_{1}^{(0)}+a_{1}^{(1)} \epsilon^{-1}+a_{1}^{(2)} \epsilon^{-2}, \\
& a_{0}(t)=a_{0}^{(0)}+a_{0}^{(1)} \epsilon^{-1}+a_{0}^{(2)} \epsilon^{-2}+a_{0}^{(3)} \epsilon^{-3} .
\end{aligned}
$$

Assuming $\epsilon \sim \delta=\epsilon$, we substitute the above approximations along with expansion given by Eq.(39) in Eq.(30). After equating the like powers of $\epsilon$ in the resultant relation, we obtain the linear dispersion relation for the system, from Eq. (30) as,

$$
\begin{gathered}
a_{3}^{(0)}\left(3 \sum_{j=0}^{N-1} S_{j}^{\prime} S_{N-1-j}^{\prime \prime}+S_{N-2}^{\prime \prime \prime}+\sum_{j=0}^{N} \sum_{l=0}^{N-j} S_{j}^{\prime} S_{l}^{\prime} S_{N-l-j}^{\prime}\right)=0, \quad N=0,1, \ldots, \infty(44) \\
+a_{2}^{(0)}\left(\sum_{j=0}^{N-1} S_{j}^{\prime} S_{N-1-j}^{\prime}+S_{N-2}^{\prime \prime}\right)+a_{2}^{(1)}\left(\sum_{j=0}^{N} S_{j}^{\prime} S_{N-j}^{\prime}+S_{N-1}^{\prime \prime}\right) \\
+a_{1}^{(0)} S_{N-2}^{\prime}+a_{1}^{(1)} S_{N-1}^{\prime}+a_{1}^{(2)} S_{N}^{\prime}+a_{0}^{(3-N)}
\end{gathered}
$$


where

$$
\begin{aligned}
a_{i}^{(j)}, S_{j}=0, \text { for } & j<0, \\
& i=0,1,2,3
\end{aligned}
$$

We note that apart from the basic assumption of well separated time-scales of our problem, two essential conditions for validity of the WKB expansion are, as $\epsilon \rightarrow 0$,

$$
\begin{aligned}
\left|\epsilon^{n} S_{n+1}(t)\right| & \ll\left|\epsilon^{n-1} S_{n}(t)\right|, \\
\left|\epsilon^{N} S_{N+1}(t)\right| & \ll 1 .
\end{aligned}
$$

We express the WKB function $S_{n}(t)$ as an integral of an unknown function $\psi_{n}(t)$,

$$
S_{n}(t) \sim \int^{t} \tau_{0}^{n-1} \psi_{n}(t) d t
$$

where $\tau_{0}$ is the shortest time-scale associated with the physical system. The WKB dispersion relation, Eq.(44) can now be examined in different regimes depending on their characteristic time-scales.

\section{B. The dust-acoustic domain $\left(\omega_{c} \sim \omega_{\varepsilon} \sim \omega_{p, n} \sim \tau_{\chi}^{-1} \ll \omega_{d}\right)$}

In the dust-acoustic domain, we assume that the characteristic time-scale is dictated by the dust-sound frequency $\omega_{d}$ which is the dominant one. It should however be noted that $\omega_{d}$ is not the usual dust-acoustic frequency which can be written as $\sqrt{T_{d} / m_{d}}$. The WKB coefficients $\underline{\underline{4}} a_{i}^{(j)}$ can now be ordered as $a_{3}=a_{3}^{(0)}, a_{2}=a_{2}^{(0)}, a_{1}=a_{1}^{(2)}+a_{1}^{(0)}$, and $a_{0}=a_{0}^{(2)}$. The second order coefficients are given by

$$
\begin{aligned}
& a_{1}^{(2)}=5 \delta_{d} Z_{d 0} \omega_{d}^{2} \\
& a_{0}^{(2)}=3 \delta_{d} Z_{d 0} \omega_{d}^{2} \Omega_{p} .
\end{aligned}
$$

To the zeroth order $(N=0)$, the dispersion relation Eq.444) now becomes

$$
\left(3 \delta_{e}+5 \tau(t)\right)\left(\omega_{0} \psi_{0}\right)^{3}+5 \delta_{d} Z_{d 0} \omega_{d}^{2}(t)\left(\omega_{0} \psi_{0}\right)=0
$$

which has two solutions for the condensation and acoustic modes respectively,

$$
\begin{aligned}
\psi_{0 \mathrm{c}} & =0 \\
\omega_{0} \psi_{0 \mathrm{~s}} & = \pm i \sqrt{\frac{a_{1}^{(2)}(t)}{a_{3}^{(0)}(t)}}= \pm i\left(\frac{5 \delta_{d} Z_{d 0} \omega_{d}^{2}(t)}{3 \delta_{e}+5 \tau(t)}\right)^{1 / 2}
\end{aligned}
$$


where $\omega_{0}=\tau_{0}^{-1}$ and the subscripts ' $\mathrm{c}$ ' and 's' refer to the condensation and sound modes.

The first order contribution $(N=1)$ to the condensation mode is given by

$$
\psi_{1 \mathrm{c}}=-\frac{3}{5} \Omega_{p}
$$

So, the condition for a growing condensation mode is given by the condition $\Omega_{p}<0$,

$$
\frac{\chi_{0} \tilde{k}^{2}}{n_{e 0}}+m_{e} T_{e 0} \frac{\partial}{\partial T_{e 0}}\left(\frac{\mathcal{L}_{e 0}}{T_{e 0}}\right)_{p_{e 0}}<0,
$$

which is similar to the condition for a static equilibrium ${ }^{3} \underline{4}$. As can be seen from the above condition (55), thermal conductivity has a stabilising effect on temperature perturbation. The corresponding critical wave number for the condensation mode is given by $\underline{\underline{4}}$,

$$
k_{c}^{2}=-\varepsilon^{2} T_{e 0} \frac{m_{e} n_{e 0}}{\chi_{0}} \frac{\partial}{\partial T_{e 0}}\left(\frac{\mathcal{L}_{e 0}}{T_{e 0}}\right)_{p_{e 0}}
$$

beyond which the condensation mode is stabilized. Note that the critical wave number has become dependent on the equilibrium electron density, which means that as more and more electrons get depleted on the surface of the massive dust particles, perturbation with longer wavelengths becomes stable $\frac{15}{}$. This is obvious as the cooling is due to the electrons, the depletion of electrons by the dust particles prevents localised regions inside a diffuse structure from effectively radiating. The dust can, however radiate at a much longer wavelength to which the medium may be opaque ${ }^{12}, 13$. Comprehensive effect of the presence of dust particles can only be realised when we consider the effect of dust-charge fluctuation in Section.V.

The first order contribution to the sound mode can be obtained from the corresponding dispersion relation as

$$
3 a_{3}^{(0)} \omega_{0}^{2}\left(\psi_{0 s} \dot{\psi}_{0 s}+\psi_{1 s} \psi_{0 s}^{2}\right)+a_{2}^{(0)}\left(\omega_{0} \psi_{0 s}\right)^{2}+a_{1}^{(2)} \psi_{1 s}+a_{0}^{(2)}=0
$$

which can be solved for $\psi_{1 s}$,

$$
\psi_{1 s}=\frac{3}{2 a_{3}^{(0)}}\left[a_{3}^{(0)}\left(2 \omega_{\varepsilon}+\omega_{c}\right)-\tau\left(\omega_{\varepsilon}+\omega_{c}\right)\right]-\frac{a_{2}^{(0)}}{2 a_{3}^{(0)}}+\frac{a_{0}^{(2)}}{2 a_{1}^{(2)}},
$$

which is real. So the existence of a growing sound mode (overstable) at $t=0$ depends on whether $\psi_{1 s}>0$ at $t=0$. 

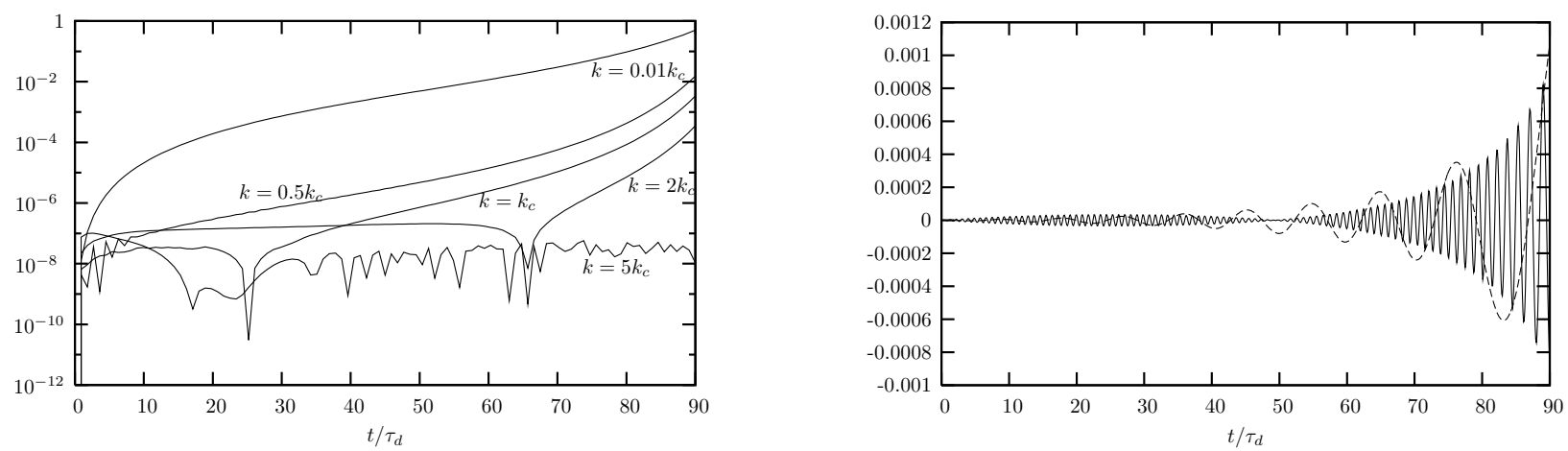

Figure 1: Comparison between the WKB and numerically computed solutions of Eq.(30) for a static equilibrium. Relative errors for different wave numbers for the condensation mode are shown in the first figure. In the second figure, relative errors for the sound mode for wave numbers $k_{0}=0.1 k_{c}$ (dashed) and $k_{0}=k_{c}$ (solid) are shown. The parameters $\alpha_{c}=0.01, \beta_{\chi}=0.5$, and $\tau_{d}=\omega_{d}^{-1}$.

\section{Validity of $W K B$ approximation}

At this point, we would like to compare the relative errors between the WKB solutions, namely (54) and (58) of the condensation and the sound modes, and the numerically computed solutions of Eq.(30). As can be seen from Fig, in the absence of expansion, the relative deviation of the WKB solutions from the numerically computed solutions is within $10^{-4}$ except for highly unstable perturbations of very long wavelengths, $k \ll k_{c}$. For short wavelength perturbation, $k>k_{c}$, the deviation is down to $10^{-8}$.

In the above analysis, we have assumed that the equilibrium heat-loss function ${ }^{33} \mathcal{L}_{e 0} \propto$ $n_{e 0} T_{e 0}^{1 / 2}$ so that the critical wave number $k_{c}$ can be expressed as

$$
\frac{k_{c}}{k_{0}}=\varepsilon\left(\frac{3 \alpha_{c}}{\beta_{\chi}}\right)^{1 / 2},
$$

where $k_{0}=\tilde{k}_{t=0}$. In the above expression, $\alpha_{c}$ and $\beta_{\chi}$ denote the ratios of the characteristic equilibrium cooling time and the thermal conduction time to the dust-acoustic time at the beginning of the expansion $(t=0)$,

$$
\alpha_{c}=\frac{\omega_{c 0}}{\omega_{0}}, \quad \beta_{\chi}=\frac{\tau_{\chi 0}^{-1}}{\omega_{0}}
$$

where $\left(\omega_{c 0}, \tau_{\chi 0}^{-1}\right)=\left(\omega_{c}, \tau_{\chi}^{-1}\right)_{t=0}$ and $\omega_{0}$ is the equilibrium dust-acoustic frequency at $t=0$,

$$
\omega_{0}^{-1}=\frac{1}{k_{0}} \sqrt{\frac{m_{d}}{T_{0}}},
$$


$T_{0}$ being the initial equilibrium electron temperature $\left[T_{e 0}\right]_{t=0}$. We would like to point out that the present form of heat-loss function inherently implies net equilibrium cooling as there is no heating source. Any non-zero equilibrium value of $\mathcal{L}_{e 0}$ actually determines the net cooling.

In working out the WKB solutions we have assumed massive dust particles ${ }^{35}$ with mass of the order of $10^{18} m_{H}, m_{H}$ being the proton mass and the dust charge number $Z_{d 0}=10^{3}$. The electron to dust ratio is fixed at $\delta_{e}=0.1$ i.e. a ninety percent depletion of the electrons on the surface of the dust particles. The initial electron temperature is assumed to be $T_{e 0}=10^{70} \mathrm{~K}$ at $t=0$. The initial equilibrium electron density $n_{e 0} \sim 10^{4} \mathrm{~cm}^{-3}$ is taken to be of the order of the average electron density in multiple-shell PNe (MSPNe) ${ }^{36}, \underline{37}$. The electron thermal conductivity at this temperature can be written as $\mathrm{s}^{33}, 34$,

$$
\chi_{0}=\frac{1.84 \times 10^{-5}}{\log \Lambda_{c}} T_{0}^{5 / 2} \operatorname{ergs~s}^{-1} \mathrm{~K}^{-1} \mathrm{~cm}^{-1},
$$

where $\log \Lambda_{c} \approx 30$ is the Coulomb logarithm. The domain of instability is defined as

$$
\omega_{c 0}<\tau_{\chi 0}^{-1}<\omega_{0},
$$

as thermal conductivity is more effective at short scale length. We have assumed a value of $\alpha_{c}=0.01$ and $\beta_{\chi}=0.5$, for which the critical wave number, given by Eq.(59), is $2.7 \times 10^{-10} \mathrm{~cm}^{-1}$ corresponding to a critical wavelength $\lambda_{c} \sim 2.3 \times 10^{10} \mathrm{~cm} \approx 7.6 \times 10^{-9} \mathrm{pc}$. As the average size of a PNe is of the order of $0.1 \mathrm{pc}^{36}$, a perturbation wavelength of the order of a few thousand kilometers $\left(\sim \lambda_{c}\right)$ is quite small compared to the size of the nebula. The characteristic time-scale $\tau_{d} \sim 10^{3}$ years, which is about $10 \%$ of the average kinematic age of a MSPNe $\underline{\underline{38}}$.

The equilibrium evolution of electron temperature can be determined from Eq.(11), which in terms of the expansion parameter $\varepsilon$ can be written as,

$$
T_{e 0}(t)=T_{0}\left[\frac{1-\left(\omega_{c 0}-\alpha\right) t}{(1+\alpha t)^{2}}\right]^{2}, \quad \omega_{c 0} t \leq 1,
$$

where $\alpha=\dot{\varepsilon}$ is the rate of expansion, which is constant in this case. The evolution of $T_{e 0}(t)$ in the absence of expansion is shown in Fig.2. Note that relation (63) is valid as long as $t<\omega_{c 0}^{-1}$. In general, equilibrium cooling should vanish as temperature approaches zero. We note that in absence of equilibrium cooling $\left(\omega_{c 0}=0\right)$, the time dependence of the equilibrium temperature can be expressed as

$$
T_{e 0}(t)=T_{0} \varepsilon(t)^{-3(\gamma-1)},
$$



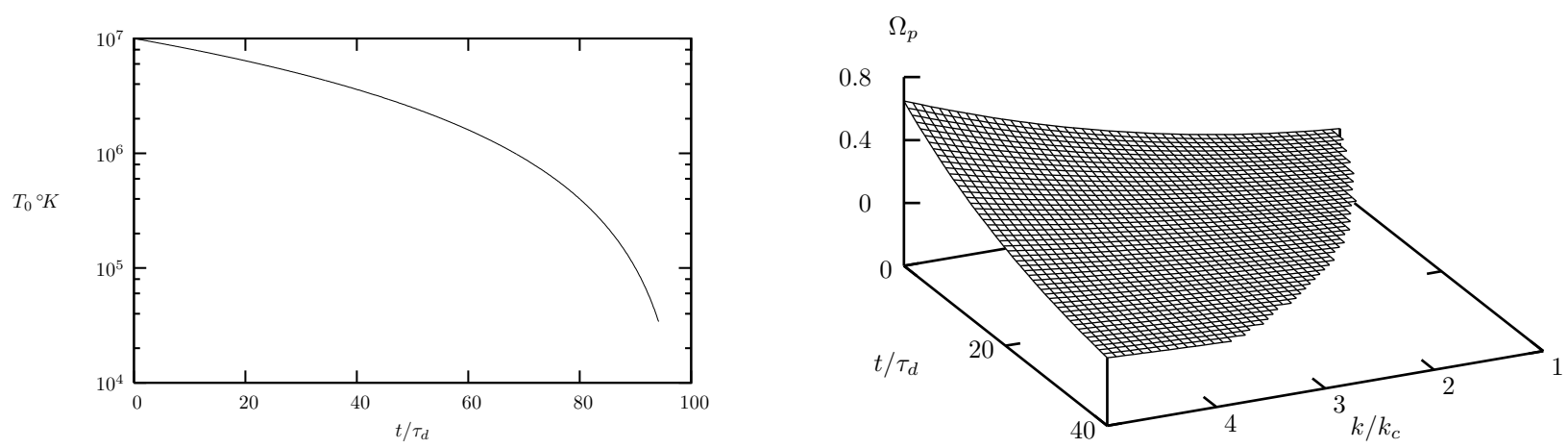

Figure 2: Equilibrium evolution of electron temperature $T_{e 0}(t)$ in absence of expansion (first diagram). The isobaric stability criterion is plotted in the second diagram against $k / k_{c}$ and $t$. For clarity, the negative values of $\Omega_{p}$ part of the $\Omega_{p}-k-t$ surface are hidden which signifies instability. $\Omega_{p}$ in the figure is normalized by the initial dust-sound frequency $\omega_{0}$.

where $\gamma$ is the ratio of specific heat, which reduces to $T_{0} \varepsilon(t)^{-2}$ for $\gamma=5 / 3$ in our case.

\section{Effect of equilibrium cooling and expansion}

Before we proceed further, a few comments and observations are to be made. If we consider only a static equilibrium with net cooling, any dependence of the equilibrium quantities is due to the evolution of equilibrium temperature. We note that as the stability (or instability) condition for the condensation mode i.e. condition (55) is valid throughout the whole time domain, the expression for critical wave number, given by Eq.(56), is dynamic due to the time dependence of the equilibrium quantities. The critical wave number is dynamically going to change, effectively determined by the detailed temperature dependence of the heatloss function $\mathcal{L}_{e 0}$. So, if we start off initially with a long wavelength stable perturbation, it might become unstable at a later time depending on the instantaneous value of the critical wave number $k_{c}$. As an example, we have shown the evolution of the quantity $\Omega_{p}$ in time with different $k / k_{c}$, in the second diagram of Fig,2, for the parameter range mentioned later in this section. As can be seen from the figure, even if the initial value for $\Omega_{p}$ at $t=0$ is positive (signifying stability for the condensation mode) for say $k \sim 2 k_{c}$, at a later time, around $t \approx 25 \tau_{d}$, the quantity falls below zero marking the onset of the instability. Similar observations can also be made for the sound mode. For the form of the heat-loss function 
$\mathcal{L}_{e 0} \propto n_{e 0} T_{e 0}^{1 / 2}$, the instability condition (55) can be written as,

$$
\beta_{\chi} \frac{\left[1-\left(\omega_{c 0}-\alpha\right) t\right]^{5}}{(1+\alpha t)^{9}} k^{2}-3 \omega_{c 0}<0,
$$

from which we see that both equilibrium cooling and expansion destabilize the condensation mode and an otherwise stable mode ultimately may become unstable due to either equilibrium cooling or expansion or both. However, the behavior of the growth rate can be ascertained only after a detailed calculation. Apparently, the instability condition for the condensation mode does not depend upon the presence of dust particles as it is just a balance between the electron conductivity and cooling due to electrons, both of which are equally affected by the dust particles.

As mentioned earlier, the existence of overstable mode depends on the positivity of $\psi_{1 s}$, given by Eq.(158), which can be simplified as,

$$
\psi_{1 s}=\left(\frac{7}{2} \omega_{\varepsilon}+6 \omega_{c}\right) \tau+\frac{3}{5}\left(\frac{1}{2} \omega_{c}-\tau_{\chi}^{-1}\right) \delta_{e} .
$$

As $\delta_{e} \leq 1$, we can write the condition of unstable acoustic mode as

$$
\frac{3}{5} \tau_{\chi}^{-1} \delta_{e}<\tau\left(\frac{7}{2} \omega_{\varepsilon}+6 \omega_{c}\right) \tau
$$

which, in the limit of vanishing background expansion $\left(\omega_{\varepsilon}=0\right)$ and $\tau \sim 1$, becomes $\tau_{\chi}^{-1} \delta_{e}<$ $10 \omega_{c}$ and for $\tau \ll 1$, reduces to $\omega_{c}>2 \tau_{\chi}^{-1}$. So, the presence of dust $\left(\delta_{e}<1\right)$ actually destabilizes the acoustic mode and a stable acoustic mode can become unstable due to the capturing of the electrons by the massive dust particles. This is due to the decrease in thermal conduction by the electrons, as more and more electrons are captured by the massive dust particles. The relation, Eq.(66) without background expansion $(\alpha=0)$, can be expressed as,

$$
\psi_{1 s}=\frac{3 \omega_{c 0}}{\left(1-\omega_{c 0} t\right)}\left(2 \tau+\frac{1}{10} \delta_{e}\right)-\frac{3}{5} \beta_{\chi}\left(1-\omega_{c 0} t\right)^{5} k^{2} \delta_{e},
$$

which shows that equilibrium cooling destabilizes the acoustic mode.

The immediate effect of equilibrium expansion is that the normal modes of the system becomes time dependent, which is analogous to change in frequency of vibration in Doppler effects. However the stability of a certain mode may depend on balancing between the opposing effects. For example, the instability condition for the acoustic mode in the limit of very small equilibrium cooling $\left(\omega_{c 0} \ll 1\right)$, can be expressed as,

$$
\frac{7 \alpha \tau}{(1+\alpha t)}>\frac{6 \beta_{\chi} k^{2} \delta_{e}}{(1+\alpha t)^{4}}
$$



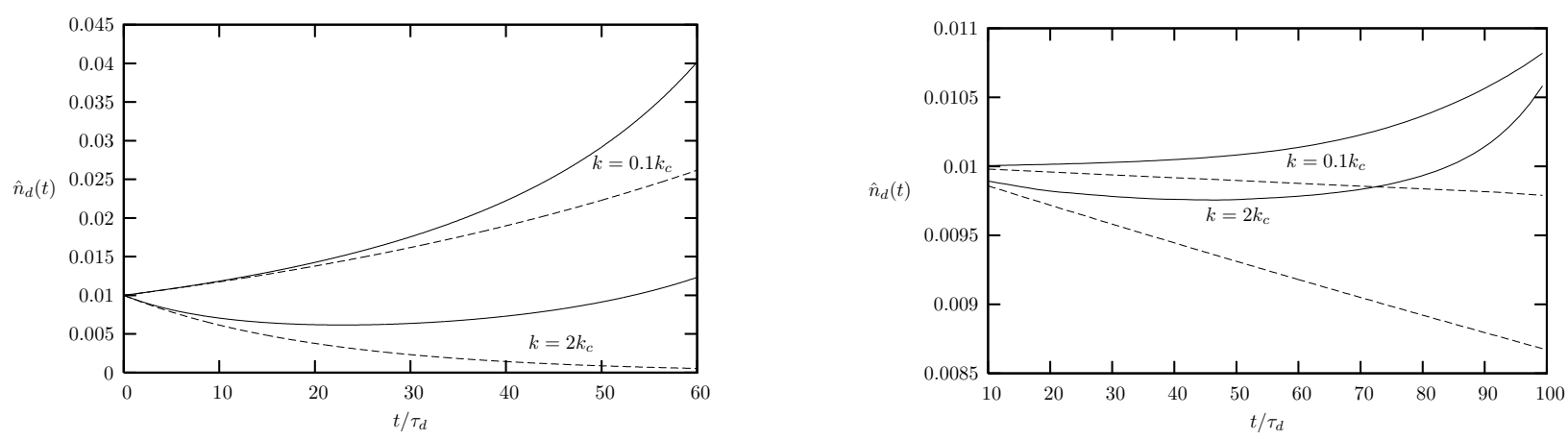

Figure 3: Comparison with the classical case ${ }^{3}$ for static equilibrium (no expansion) in presence of equilibrium temperature evolution for the perturbed dust density for the condensation mode (first figure) and wave mode (second figure). Rest of the parameters are same as for Fig,2, The dashed lines indicate the classical Field's behavior. The values of wave number are shown is at $t=0$.

and we see that background expansion too destabilizes the acoustic mode. Physically, expansion nullifies the effect of thermal conductivity as thermal conductivity is most effective at short scale lengths, leaving the onset of thermal instability entirely on the isobaric cooling.

The cumulative effect of equilibrium cooling, background expansion, and electron capturing my massive dust particles is to destabilize the condensation and the acoustic modes which effectively increases (reduces) the cut-off wavenumber (wavelength) beyond which the modes are stable. Therefore, we conclude that as the nebulae evolve due to expansion, the microstructures eventually die out and the presence of dust particles enhances this effect, which actually conforms to the observational studies, which report that the microstructures are transient compared to the evolutionary life (due to expansion) of the planetary nebulae $\mathrm{e}^{29}$.

We now compute the WKB solutions of the normalized perturbed dust density $\hat{n}_{d}(t)$ for the parameter range discussed above. The evolution of the perturbed dust density with only equilibrium cooling for the condensation mode and the acoustic mode are shown in Fig 3 , As can be seen from the figures, though the classical condensation mode is stable beyond the critical wave number $k_{c}$, in presence of equilibrium cooling, the condensation mode can grow further. However, for $k \gg k_{c}$, the mode is stabilized. The equilibrium cooling has similar effects on the acoustic mode (see the second figure in Fig[3). The classical results with no equilibrium expansion and cooling ${ }^{3}$ are shown as dashed curves. The initial level of perturbation is fixed at 0.01 in all the cases.

In Fig, 4, we show the effect of equilibrium expansion on the modes. The rate of equilib- 

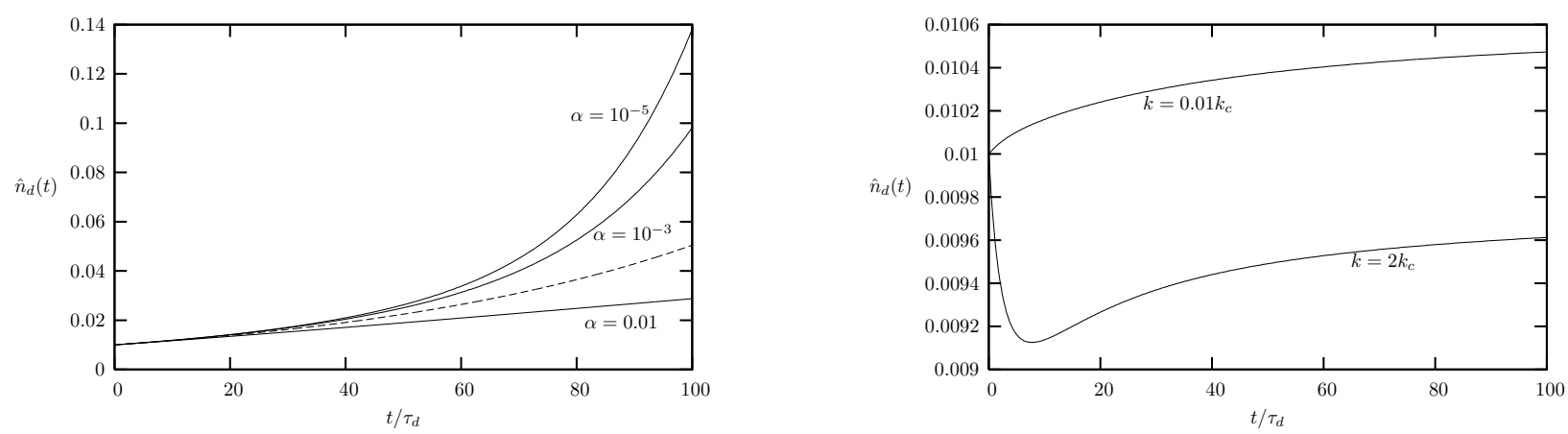

Figure 4: In the first diagram the comparison of the WKB solutions for the condensation mode with the classical Field's case in presence of equilibrium expansion and net equilibrium cooling, is shown for $k=0.01 \varepsilon k_{c}$. Other parameters are same as before and the dotted line shows the prediction of the classical theory. In the second diagram, the destabilisation of the condensation mode for rapid expansion $(\alpha=0.1)$ is shown for different $k$.

rium expansion is given by $\alpha$. As seen from the first diagram of Fig, 4, rapid expansion causes the growth rate of the condensation mode to decrease in the initial phase. However, finally, expansion destabilises an otherwise stable mode, which is shown in the second diagram of Fig 4, where we have set the expansion rate at $\sim 10 \%$ of the dust-acoustic frequency.

\section{The heat-conduction domain $\left(\omega_{c}, \omega_{\varepsilon}, \omega_{p, n}, \omega_{d} \ll \tau_{\chi}^{-1}\right)$}

At very small spatial scale, the heat conduction through the electrons dominates and the thermal equilibration of the electrons prevents any thermal instability. With this ordering, the WKB expansion yields one condensation mode

$$
\omega_{0} \psi_{0 \mathrm{c}}=-\frac{3 \tau_{\chi}^{-1}\left(\tau+\delta_{e}\right)}{\left(3 \delta_{e}+5 \tau\right)},
$$

which is stable.

\section{DUST CHARGE FLUCTUATION : NUMERICAL SOLUTION}

In this section we let the dust charge to change according to the dust charging equation Eq.(26). In what follows, we however neglect the natural decay rate of charge fluctuations 

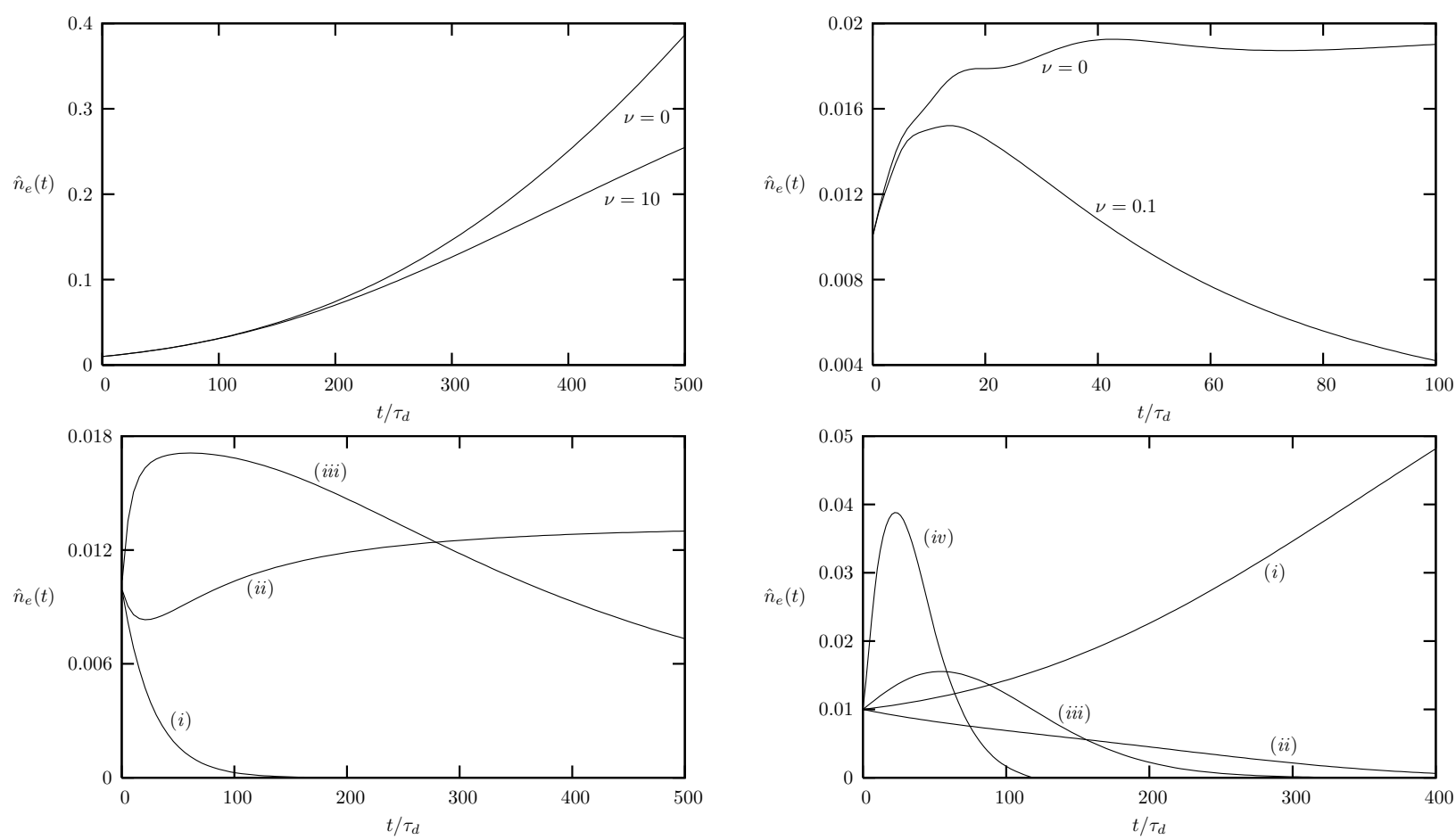

Figure 5: Row-wise from the top - (a) Low-frequency response of condensation mode for low cooling and expansion, $\gamma_{c}-\gamma_{h}=10^{-3}, \alpha=10^{-3}$ and $(b)$ high cooling and expansion, $\gamma_{c}-\gamma_{h}=$ 0.1, $\alpha=0.1$. (c) High frequency response to the condensation mode $(i) \alpha=0, \gamma_{c}-\gamma_{h}=0, \nu=0.1$, (ii) $\alpha=0.01, \gamma_{c}-\gamma_{h}=0.01, \nu=0.1,($ iii $) \alpha=0.1, \gamma_{c}-\gamma_{h}=0.1, \nu=0.1$. (d) Low frequency response to the sound mode $(i) \alpha=10^{-3}, \gamma_{c}-\gamma_{h}=10^{-3}, \nu=0,(i i) \alpha=10^{-3}, \gamma_{c}-\gamma_{h}=10^{-3}, \nu=$ 0.01, $($ iii $) \alpha=0.01, \gamma_{c}-\gamma_{h}=0.01, \nu=0.01,($ iv $) \alpha=0.1, \gamma_{c}-\gamma_{h}=0.1, \nu=0.01$.

$\eta$. Using Eqs.(16) and (15) we can write the charging equation as

$$
\frac{d \hat{Z}_{d}}{d t}=\nu\left(-\tau \hat{p}_{e}-\hat{n}_{e}\right)
$$

where $\hat{Z}_{d}$ is normalized perturbed dust-charge number and $\nu$ is the charge fluctuation frequency normalized to initial dust-acoustic frequency $\omega_{0}$. The fluctuation of dust charge introduces another time-scale into the problem, which we would like to keep at an arbitrary level. Therefore a clear separation of time-scales is not possible and we investigate the dispersion relation numerically instead of applying WKB approximation.

In order to facilitate equilibrium balance between radiation loss and heating, we assume a form of the net cooling function with both heating and cooling having same dependence on density ${ }^{3}$,

$$
\mathcal{L}_{e 0}=f_{1}\left(n_{e 0}\right)\left[f_{2}\left(T_{e 0}\right)-f_{3}\left(T_{e 0}\right)\right]
$$


and the equilibrium temperature $T_{0}$ is determined by the condition $\mathcal{L}_{e 0}=0$. With the heatloss function given by Eq.(72), the isobaric stability condition becomes $f_{2}^{\prime}\left(T_{e 0}\right)>f_{3}^{\prime}\left(T_{e 0}\right)$. Without loss of any generality, we assume that $f_{2} \propto T_{e 0}, f_{3} \propto T_{e 0}^{2}$, and $f_{1} \propto n_{e 0}$ so that the net heat-loss function can be written as,

$$
\mathcal{L}_{e 0}=n_{e 0}\left(C_{1} T_{e 0}-C_{2} T_{e 0}^{2}\right)=\Lambda-\Gamma
$$

where $C_{1,2}$ are proportionality constants and $\Lambda$ and $\Gamma$ are equivalent equilibrium cooling and heating. The cooling time-scale in terms of $\omega_{c 0}$ can be written as

$$
\omega_{c 0}=\omega_{0} \frac{n_{e 0}}{n_{0}}\left(\gamma_{c}-\gamma_{h} \frac{T_{e 0}}{T_{0}}\right)
$$

with $\gamma_{c, h}$ being the normalised cooling and heating functions and $n_{0}=\left.n_{e 0}\right|_{t=0}$.

The comprehensive effect of dust-charge fluctuation on the perturbation amplitude is shown in Fig.5. In the top panel of Fig 15 , the low-frequency response of the condensation mode is shown for low cooling and slow expansion (first diagram) and high cooling and rapid expansion (second diagram). Though the effect of charge-fluctuation is to stabilize the mode, the effect is more pronounced in the case of rapid expansion as can be seen from the level of perturbation amplitude. For charge fluctuation frequency as low as $10 \%$ of the dust-acoustic frequency, the condensation mode is completely stabilized beyond $20 \tau_{d}$. As background expansion tends to enhance the effect of cooling, we have assumed the cooling and expansion time-scales to be of the same order. The high-frequency response of the condensation mode is shown in the first diagram of the lower panel of Fig 5 where the effect of dust-charge fluctuation is shown for different rates of cooling and expansion. We observe that with finite dust-charge fluctuation, expansion and equilibrium cooling actually destabilise the condensation mode [curve $(i i)$ in Fig 5(5)]. However rapid expansion leads to stabilisation. This is a distinct departure from the behaviour of the mode in case of low-frequency perturbation.

The behaviour of the acoustic mode with low-frequency perturbation is shown in the second figure of the lower panel of Fig,5. Increasing equilibrium cooling and expansion tend to stabilise the acoustic mode with finite dust-charge fluctuation. High-frequency perturbations of the acoustic mode are always damped. 


\section{CONCLUSION}

We have considered the the role of thermal instability of an ionized plasma with an expanding equilibrium and net equilibrium cooling, which has a considerable presence of dust and discussed the outcome of the analysis in the context of expanding planetary nebulae. We have found that the cumulative effect of equilibrium cooling, background expansion and the electron capturing by the massive dust particles is to destabilize the condensation and the acoustic modes which reduces the cut-off wavelengths beyond which the modes are stable. Therefore, we see that as a planetary nebula evolves due to expansion, the microstructures eventually die out, which is in agreement with the observational reports that these microstructures are transient compared to the evolutionary life of a nebula. We have shown that the presence of dust particles in this ionized environment tends to destabilize the acoustic mode.

However, the fluctuation of dust-charge severely damps these instabilities and the modes are completely stabilized for sufficiently large fluctuation frequency. As there are no conclusive report on the dust-charge fluctuation parameter in these ionized plasmas, it will be too much far fetched to say that the dust-charge fluctuation completely suppresses the formation of the microstructures, which can be formed out of radiation induced instabilities. It will be of much interest to view these results with newer experimental data.

\section{Appendix}

In this appendix, we show that for non-static equilibrium, it is essential for the normal mode of the system to be expressed exclusively as a function of time and a decomposition 32 of the kind expressed in Eq.(29) is not fruitful. As an example, we take the case represented in Fig. 1 , for $k=0.1 k_{c}$ for condensation modes and $k=5 k_{c}$ for acoustic modes. We consider, only the effect of equilibrium expansion in the limit of no net equilibrium cooling. Thus the equilibrium evolution of the temperature is due to expansion only [see Eq.(11)]. In Fig.6, we show the growth of the perturbed dust density $\hat{n}_{d}(t)$ as obtained by the full numerical solution of Eqs.(14)-(16), (22), (24), (26), and (27) and the solution through the WKB expansion, Eq.(44). The solution, as given by the prescription represented in Eq.(29) is plotted in the figures as dashed lines. As can be seen, the solution from the normal mode 

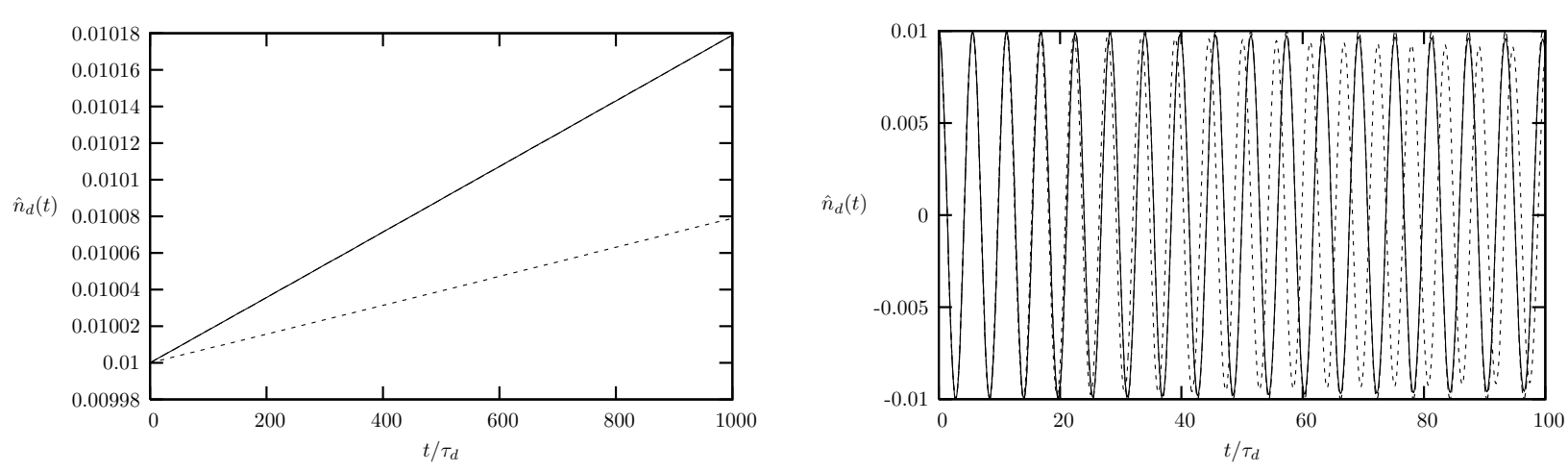

Figure 6: The numerical solution of Eqs.(14)-(16), (22), (24), (26), and (27), WKB solution, and the decomposed solution (shown as dashed lines). The first figure is for condensation mode and the second one is for wave mode. The solid curves represent the numerical and WKB solutions which are almost indistinguishable. In the same parameter regime, the decomposed solution has a large deviation in amplitudes and phases.

decomposition has a large deviation from the exact numerical solution, in terms of growth rates, whereas, the WKB solution almost coincides with the numerical solution. Also note the difference in phases of the wave mode solution from the numerical one, while the WKB solution has been able to keep in phase with the numerical solution.

1 E. N. Parker, Astrophys. J. 117, 431 (1953).

2 R. Weymann, Astrophys. J. 132, 452 (1960).

3 G. B. Field, Astrophys. J. 142, 531 (1965).

4 A. J. Gomez-Pelaez and F. Moreno-Insertis, Astrophys. J. 569, 766 (2002).

5 C. D. C. Steele, M. H. Ibáñez, and E. Sira, Phys. Plasmas 7, 3781 (2000).

6 C. H. Baek, H. Kang, J. Kim, and D. Ryu, Astrophys. J. 630, 689 (2005).

7 C. H. Baek, D. Ryu, H. Kang, and J. Kim, Astrophys. J. 643, L83 (2006).

8 R. A. Piontek and E. C. Ostriker, Astrophys. J. 601, 905 (2004).

9 R. A. Piontek and E. C. Ostriker, Astrophys. J. 629, 849 (2005).

10 J. C. Shields and R. C. Kennicutt, Astrophys. J. 454, 807 (1995).

11 M. A. Dopita and R. S. Sutherland, Astrophys. J. 539, 742 (2000).

12 S. Oliveria and W. J. Maciel, Astrophys. Sp. Sc. 126, 211 (1986). 
13 J. A. Baldwin, et. al., Astrophys. J. 374, 580 (1991).

14 M. R. Jana, A. Sen, and P. K. Kaw, Phys. Rev. E 48, 3930 (1993).

15 M. P. Bora, Phys. Plasmas 11, 523 (2004).

16 J. P. Phillips, Astron. Astrophys. 393, 1027 (2002).

17 D. Schönberner, et. al., Astron. Astrophys. 431, 963 (2005).

18 G. Stasinska and R. Szczerba, arXiv astro-ph/9911006 v1 (2006).

19 S. R. Pottasch, Planetary Nebulae (D. Reidel Publ. Comp. 1984).

20 P. Lenzuni, et. al., Astrophys. J. 345, 306 (1989).

21 J. Kingdon, G. J. Ferland, and W. A. Fiebelman, Astrophys. J. 439, 793 (1995).

22 J. Kingdon and G. J. Ferland, Astrophys. J. 477, 732 (1997).

23 P. A. M. van Hoof, et. al., Astrophys. J. 532, 384 (2000).

24 K. Volk, H. Dinerstein, and C. Sneden, in Planetary Nebuae, Proc. IAU Symp. 180, p. 284, 1997.

25 G. B. Field, Astrophys. J. 187, 453 (1974).

26 V. A. Okorokov et. al., Astron. Astrophys. 142, 441 (1985).

27 H. Marten, R. Szczerba, and Th. Blöcker, in Planetary Nebuae (Proc. IAU Symp. 155, 1993), p. 363 .

28 B. P. Pandey, V. Krishan, and M. Roy, Pramana 56, 95 (2001).

29 B. Balick et. al., Astrophys. J. 424, 800 (1994).

30 S. Weinberg, Gravitation and Cosmology: Principles and Applications of the General Theory of Relativity (Wiley, 2001).

31 C. M. Bender and S. A. Orszag, Advanced Mathematical Methods for Scientists and Engineers (McGraw Hill, New York, 1978).

32 M. Nejad-Asghar and J. Ghanbari, arXiv:astro-ph/0603172 v2 (2006).

33 L. Spitzer, Physics of Fully Ionized Gases (Wiley, New York, 1962).

34 G. B. Rybicki and A. P. Lightman, Radiative Precesses in Astrophysics (Wiley, New York, 1979).

35 D. A. Mendis and M. Rosenberg, Ann. Rev. Astron. Astrophys. 32, 419 (1994).

36 M. A. Guerrero, E. Villaver, and A. Manchado, Astrophys. J. 507, 889 (1998).

37 L. Stanghellini and J. B. Kaler, Astrophys. J. 343, 811 (1995).

38 Y.-H. Chou, in Planetary Nebulae, edited by S. Torres-Peimbert (IAU Symp. 131, Kluwer, 
Dordrecht, 1989), p. 105. 